\title{
BACK TO RS-SR SPECTRAL THEORY
}

\author{
C. BENHIDA \\ Université de Lille 1, UFR de Mathématiques \\ CNRS-UMR 8524, Bât. M2, 59655 Villeneuve Cedex, France \\ E-mail: benhida@math.univ-lille1.fr \\ E. H. ZEROUALI \\ Faculté des Sciences de Rabat, BP 1014 Rabat, Morocco \\ E-mail: zerouali@fsr.ac.ma
}

\begin{abstract}
Let $X, Y$ be Banach spaces, $S: X \rightarrow Y$ and $R: Y \rightarrow X$ be bounded operators. We investigate common spectral properties of $R S$ and $S R$. We then apply the result obtained to extensions, Aluthge transforms and upper triangular operator matrices.
\end{abstract}

1. Introduction. Let $X$ and $Y$ be Banach spaces and let $\mathcal{L}(X, Y)$ denote the space of all bounded linear operators from $X$ to $Y$. For a bounded linear operator $T \in \mathcal{L}(X)=$ : $\mathcal{L}(X, X)$, let $\sigma(T)$ denote the spectrum, $R(T)$ its range space and let $N(T)$ denote the null space.

A complex number $\lambda$ is said to be in the local resolvent set $\rho_{T}(x)$ of $T$ at $x \in X$ if there exists an analytic $X$-valued function $f$ on some open neighbourhood $U$ of $\lambda$ such that $(T-\mu) f(\mu)=x \quad$ for all $\mu \in U$.

The local spectrum of $T$ at $x$ is then the set $\sigma_{T}(x)=\mathbb{C} \backslash \rho_{T}(x)$. It is immediate that $\sigma_{T}(x)$ is a closed subset of $\sigma(T)$. However, the local spectrum can be the empty set. This fact is related to the single valued extension property. To be precise, let $D(\lambda, r)$ be the open disc centered at $\lambda \in \mathbb{C}$ and with radius $r>0$, the corresponding closed disc will be denoted by $\bar{D}(\lambda, r)$. We will say that $T$ has the single valued extension property (or $S V E P)$ at $\lambda \in \mathbb{C}$ if there exists $r>0$ such that for every open subset $U \subset D(\lambda, r)$, the constant function $f \equiv 0$ is the only analytic solution of the equation $(T-\mu) f(\mu)=0$. We set $\mathcal{S}(T)$, for the open set where $T$ fails to have the $S V E P$. An operator $T$ is said to have the $S V E P$ precisely when $\mathcal{S}(T)=\emptyset$. $S V E P$ may also be characterized using local

2000 Mathematics Subject Classification: Primary 47A11, 47A10.

Key words and phrases: local spectral theory, linear operators, extensions, Aluthge transform. The paper is in final form and no version of it will be published elsewhere. 
spectra in the following sense: $T$ has the $S V E P$ if and only if $\sigma_{T}(x) \neq \emptyset$ for every $x \neq 0$. See $[14,22]$.

It is known that for every bounded operator $T \in \mathcal{L}(X)$ we have

$$
\sigma(T)=\bigcup_{x \in X} \sigma_{T}(x) \cup \mathcal{S}(T)
$$

Thus if $T$ has the $S V E P$, we get $\sigma(T)=\bigcup_{x \in X} \sigma_{T}(x)$.

Another important property in local spectral theory is Bishop's property ( $\beta)$. The operator $T$ satisfies Bishop's property $(\beta)$ at $\lambda \in \mathbb{C}$ if there exists $r>0$ such that for every open subset $U \subset D(\lambda, r)$ and for any sequence $\left(f_{n}\right)_{n}$ of analytic $X$-valued functions on $U$ with $(T-\mu) f_{n}(\mu) \rightarrow 0$ as $n \rightarrow \infty$ uniformly on compact subsets of $U$, we have $f_{n}(\mu) \rightarrow 0$ as $n \rightarrow \infty$ uniformly on compact subsets of $U$. We denote by $\sigma_{\beta}(T)$ the set where $T$ fails to satisfy $(\beta)$ and we say that $T$ satisfies Bishop's property $(\beta)$ if $\sigma_{\beta}(T)=\emptyset$. For more details, see [22].

The operator $T$ is said to have the decomposition property $(\delta)$ if for every finite open cover $\left\{U_{1}, \ldots, U_{n}\right\}$ of $\mathbb{C}$,

$$
\mathcal{X}_{T}\left(\bar{U}_{1}\right)+\cdots+\mathcal{X}_{T}\left(\bar{U}_{n}\right)=X,
$$

where $\mathcal{X}_{T}(F)$ is the glocal analytic spectral subspace associated with $F$ and defined to be as the set of all elements $x \in X$ such that the equation $(T-\lambda) x(\lambda)=x$ has a global analytic solution on $\mathbb{C} \backslash F$.

The properties $(\beta)$ and $(\delta)$ are dual to each other in the sense that $T$ has $(\delta)$ if and only if $T^{*}$ satisfies $(\beta)$ and $T$ has $(\beta)$ if and only if $T^{*}$ satisfies $(\delta)$. A weaker version of property $(\delta)$ is $\left(\delta_{w}\right)$ introduced in [30] as follows:

An operator $T$ has the weak decomposition property $\left(\delta_{w}\right)$ if for every finite open cover $\left\{U_{1}, \ldots, U_{n}\right\}$ of $\mathbb{C}$ we have

$$
\mathcal{X}_{T}\left(\bar{U}_{1}\right)+\cdots+\mathcal{X}_{T}\left(\bar{U}_{n}\right) \text { is dense in } X .
$$

A bounded linear operator $T$ on a Banach space $X$ is said to be decomposable provided that for every finite open cover $\left\{U_{1}, \ldots, U_{n}\right\}$ of $\mathbb{C}$, there exist $X_{1}, \ldots, X_{n}$ closed $T$ invariant subspaces of $X$ such that

$$
\sigma\left(T \mid X_{i}\right) \subseteq U_{i} \quad \text { for } i=1, \ldots, n \text { and } X_{1}+\cdots+X_{n}=X .
$$

The class of decomposable operators includes all normal operators and more generally all spectral operators. Operators with totally disconnected spectrum are decomposable by the Riesz functional calculus. In particular, compact and algebraic operators are decomposable.

It is also known that $(\beta)$ characterizes operators with decomposable extensions and in particular operators with $(\beta)$ are provided by isometries and subnormal operators [2]. The property $(\beta)$ is hence conserved by restrictions while $(\delta)$ is transferred to quotient operators. We refer to $[1,2,22]$ for a complete study and further properties and results.

In this paper, our aim is to show that if $S: X \rightarrow Y$ and $R: Y \rightarrow X$ are bounded linear operators then $S R$ and $R S$ share many of their local spectral properties. That is, $S R$ belongs to any of the previous classes if and only if $R S$ does. 
The results obtained here apply to upper block operator matrices. To be more precise, for $A \in \mathcal{L}(X), B \in \mathcal{L}(Y)$ and $C \in \mathcal{L}(Y, X)$, let $M_{C}$ be the upper triangular $2 \times 2$ matrix $\left[\begin{array}{ll}A & C \\ 0 & B\end{array}\right]$. Then for which $C$ do we have $\Sigma\left(M_{C}\right)=\Sigma(A) \cup \Sigma(B)$, where $\Sigma$ runs over different spectra?

It is proved in section 2 that $S R$ has the single valued extension property (resp. Bishop's property $(\beta)$; resp. spectral decomposition property $(\delta))$ if and only if $R S$ has the single valued extension property (resp. Bishop's property $(\beta)$; resp. spectral decomposition property $(\delta)$ ). We also show that $R S$ is subscalar if and only if $S R$ is subscalar. The latter result extends a previous one in [9] given for injective operators.

We devote section 3 to the connections between local spectra, spectral subspaces and related properties of $R S$ and $S R$.

We give in section 4 some applications to Aluthge transforms of operators and to operator matrices.

2. Local spectral theory for $R S$ and $S R$. Throughout this section, $S: X \rightarrow Y$ and $R: Y \rightarrow X$ are bounded linear operators and for the obvious symmetry reason, we shall show, in the proofs, only one way any time we find an equivalence statement.

Proposition 2.1. Let $\lambda \in \mathbb{C}$ be a complex number, then $R S$ has the $S V E P$ at $\lambda$ if and only if $S R$ has the $S V E P$ at $\lambda$. In particular $R S$ has the $S V E P$ if and only if $S R$ has the $S V E P$.

Proof. Suppose that $R S$ has the $S V E P$ at $\lambda$ and let $x(\mu)$ be an $Y$-valued analytic function in a neighbourhood $V(\lambda)$ of $\lambda$ such that

$$
(S R-\mu) x(\mu)=0(\mu \in V) .
$$

If we take $R$ values in equality (4), we obtain $(R S-\mu) R x(\mu)=0$. Now, since $R$ is a bounded linear operator, $R x(\mu)$ is an $X$-valued analytic function in $V(\lambda)$. Thus $R x(\mu)=0$ and Equation (4) implies that $\mu x(\mu)=0$. So $x(\mu)=0$ and $S R$ has the $S V E P$ property at $\lambda$.

We extend now [10, Theorem 5] by removing injectivity of $R$ and $S$.

Proposition 2.2. Let $R$ and $S$ be bounded operators, then

$$
\sigma_{\beta}(R S)=\sigma_{\beta}(S R) .
$$

In particular $S R$ satisfies Bishop's property $(\beta)$ (resp. $(\delta))$ if and only if $R S$ satisfies Bishop's property $(\beta)$ (resp. $(\delta)$ ).

Proof. Let $\lambda \in \mathbb{C} \backslash \sigma_{\beta}(R S)$ and let $\left(f_{n}\right)_{n}$ be a sequence of $Y$-valued analytic functions in a neighbourhood of $\lambda$ such that

$$
\lim _{n \rightarrow+\infty}(S R-\mu) f_{n}(\mu)=0 \quad \text { in } \mathcal{O}(V(\lambda), Y) .
$$

By taking $R$, we obtain

$$
\lim _{n \rightarrow+\infty}(R S-\mu) R f_{n}(\mu)=0 \quad \text { in } \mathcal{O}(V(\lambda), X) .
$$

Also $R f_{n}(.) \in \mathcal{O}(V(\lambda), X)$ since $R$ is a bounded linear operator. 
So $R f_{n}(\mu)$ converges to 0 on compact sets and by taking $S$ values, $S R f_{n}(\mu)$ converges to 0 on compact sets.

From Equation (5), we get $\left(\mu f_{n}(\mu)\right)_{n}$ converges to 0 on compact sets. Now since $f_{n}$ are analytic, the maximum modulus principle implies that $\left(f_{n}\right)_{n}$ converges to 0 on compact sets. Thus $\lambda \in \mathbb{C} \backslash \sigma_{\beta}(S R)$.

Property $(\delta)$ is obtained by passing to duals.

REMARK 2.1. We notice here that if we set $S R=T$ and $R S=Q$, then $T$ and $Q$ are intertwined in the following way, $R T=Q R$ and $T S=S Q$. Hence from [22] it follows that if $R$ and $S$ are onto, then $R S$ has $(\delta)$ if and only if $S R$ has $(\delta)$.

Since an operator is decomposable if and only if it has both $(\beta)$ and $(\delta)$, we obtain COROllary 2.1. RS is decomposable if and only if $S R$ is decomposable.

The class of decomposable operators includes many other interesting classes as normal operators of spectral operators. Similarly the class of operators with $(\beta)$ includes subnormals and hyponormals.

In view of Corollary 2.1, one may expect that $R S$ is normal (subnormal or hyponormal) if and only if $S R$ is. We provide here two examples disproving this fact.

To get an example with $S R$ hyponormal but not $R S$ it suffices to consider any nonhyponormal operator such that its Aluthge transform is hyponormal. Now as observed is section 4, properties of Aluthge transforms are derived from the $R S$ - $S R$ phenomena.

To deal with subnormality let $H^{2}$ be Hilbert space and $\left\{e_{n}, n \geq 0\right\}$ be any orthonormal basis. We consider $S$ for the weighted shift $S e_{n}=(n+2) e_{n+1}$ and for $R$ the diagonal operator $R e_{n}=\frac{1}{n+1} e_{n}$. Then $R S e_{n}=e_{n+1}$ and $S R e_{n}=\frac{n+2}{n+1} e_{n+1}$. In particular $R S$ is subnormal while $S R$ is not hyponormal since the associated weight is not increasing.

It is then natural to ask to which classes proposition 2.2 and corollaries 2.1 can be extended.

Some other properties are also shared by $R S$ and $S R$, for example

Proposition 2.3. We have

- $R S$ is nilpotent if and only if $S R$ is,

- $R S$ is quasi-nilpotent if and only if $S R$ is,

- $R S$ is compact if and only if $S R$ is.

Proof. The first two assertions follow from the identity $(R S)^{n}=R(S R)^{n-1} S$. The third assertion can be found in [5].

An operator $T$ is said to be generalized scalar if there exists a continuous algebra homomorphism $\Phi: \mathcal{E}(\mathbb{C}) \rightarrow \mathcal{L}(X)$ with $\Phi(1)=I$ and $\Phi(z)=T$. Here $\mathcal{E}(\mathbb{C})$ denotes the algebra of all infinitely differentiable functions on $\mathbb{C}$ endowed with the topology of uniform convergence on compact sets of such functions and their derivatives. Let $\mathcal{U}$ be an open subset of the complex plane and $\mathcal{E}(\mathcal{U})$ be the Fréchet algebra of all infinitely differentiable functions on $\mathcal{U}$ endowed with the topology of uniform convergence on compact subsets of $\mathcal{U}$ of all derivatives. The operator $T$ is said to have property $(\beta)_{\epsilon}$ at $\lambda$ if there exists $\mathcal{U}$ a neighbourhood of $\lambda$ such that for each open set $O \subset \mathcal{U}$ and for any sequence $\left(f_{n}\right)_{n}$ of $X$-valued functions in $\mathcal{E}(O, X)$ the convergence of $(T-z) f_{n}(z)$ to zero in $\mathcal{E}(O, X)$ 
implies the convergence of $f_{n}$ to zero in $\mathcal{E}(O, X)$. Denote by $\sigma_{(\beta)_{\epsilon}}(T)$ the set where $T$ fails to satisfy $(\beta)_{\epsilon}$. We will say that $T$ satisfies property $(\beta)_{\epsilon}$ if $\sigma_{(\beta)_{\epsilon}}(T)=\emptyset$. It is not hard to see that $\lambda \notin \sigma_{(\beta)_{\epsilon}}(T)$ if and only of there exists $\mathcal{U}$ a neighbourhood of $\lambda$ such that $T_{U}: f \rightarrow(z \mapsto(T-z) f(z))$ is one to one with closed range on $\mathcal{E}(O, X)$. It is also known that property $(\beta)_{\epsilon}$ characterizes those operators with some generalized scalar extension. Such operators are called subscalar. We have

THEOREM 2.1.

$$
\sigma_{(\beta)_{\epsilon}}(R S)=\sigma_{(\beta)_{\epsilon}}(S R)
$$

Obviously operators with property $(\beta)_{\epsilon}$ have Bishop's property $(\beta)$. However, a direct adaptation of the proof of Proposition 2.2 will not work since the maximum modulus principle is no more valid for infinitely differentiable functions. To avoid this difficulty the following lemma given in [8] provides an alternative way.

LEMMA 2.1. Let $O$ be an open set and $\left(f_{n}\right)_{n}$ be a sequence in $\mathcal{E}(O, X)$ such that $\left(z f_{n}(z)\right)_{n}$ converges to zero in $\mathcal{E}(O, X)$. Then $\left(f_{n}\right)_{n}$ converges to zero in $\mathcal{E}(O, X)$.

Suppose now $\lambda \notin \sigma_{(\beta)_{\epsilon}}(R S)$ and let $O$ be a neighbourhood of $\lambda$ such that $O \cap$ $\sigma_{(\beta)_{\epsilon}}(R S)=\emptyset$. If $\left(f_{n}\right)_{n}$ is a sequence in $\mathcal{E}(O, X)$ such that $(R S-z) f_{n}(z)$ converges to zero in $\mathcal{E}(O, X)$, then $\lim _{n \rightarrow \infty}(S R-z) S f_{n}(z)=0$ and hence $\lim _{n \rightarrow \infty} S f_{n}(z)=0$. It follows that $\left(z f_{n}(z)\right)_{n}$ converges to zero in $\mathcal{E}(O, X)$. By lemma $2.1,\left(f_{n}\right)_{n}$ converges to zero in $\mathcal{E}(O, X)$. Finally $\lambda \notin \sigma_{(\beta)_{\epsilon}}(S R)$. The reverse implication is obtained by symmetry.

We deduce the following corollary:

COROLlary 2.2. $R S$ is subscalar if and only if $S R$ is subscalar.

The previous corollary is given by Lin Chen et al. [9, Theorem A] under the extra assumption that $R$ and $S$ are one to one.

3. Local spectra for $R S$ and $S R$. In this section we investigate the link between local spectra of $R S$ and $S R$. We first give the following proposition from [7]:

Proposition 3.1. Let $R$ and $S$ be as above. For every $x \in X$, we have

i) $\sigma_{S R}(S x) \subset \sigma_{R S}(x) \subset \sigma_{S R}(S x) \cup\{0\}\left(\operatorname{resp} . \sigma_{R S}(R y) \subset \sigma_{S R}(y) \subset \sigma_{R S}(R y) \cup\{0\}\right.$ for every $y \in Y)$.

ii) If moreover $S$ is one to one, $\sigma_{R S}(x)=\sigma_{S R}(S x)$ (resp. if $R$ is one to one $\sigma_{R S}(R y)=$ $\sigma_{S R}(y)$ for every $\left.y \in Y\right)$.

The example below shows that if $S$ is not one to one, it may happen that $\sigma_{R S}(x) \neq$ $\sigma_{S R}(S x)$.

EXAMPLE 3.1. Let $S$ be the shift operator defined on the usual Hardy space and let $R=S^{*}$ be its adjoint operator. Then $R S$ is the identity operator, while $S R$ is the projection operator on $R(S)$. In particular $\sigma_{R S}(x)=\{1\}$, for every nonzero $x \in H$, $\sigma_{S R}(x)=\{1\}$ if $x \in R(S), \sigma_{S R}(x)=\{0\}$ if $x \in N(R)$ and $\sigma_{S R}(x)=\{0,1\}$ otherwise. The inclusion $\sigma_{R S}(S x) \subset \sigma_{S R}(x)$ is then strict in the last case.

It is known that $\sigma(R S) \backslash\{0\}=\sigma(S R) \backslash\{0\}$ and the previous example shows that the equality $\sigma(R S)=\sigma(S R)$ is not true in general. The latter equality has been proved 
to be true in the case where $R$ (or $S$ ) is normal, see [11]. As a immediate application of Proposition 3.1, we obtain

Corollary 3.1. Let $R$ and $S$ be as above, then $\sigma(R S)=\sigma(S R)$ in the following cases:

(1) $S$ and $R$ are injective.

(2) $S$ and $S^{*}$ are not injective.

(3) $S$ and $R$ are not injective.

(4) $S$ or $R$ is injective with dense range.

Proof. If $S$ is injective, then

$$
\begin{array}{cc}
\sigma(R S) & \sigma(S R) \\
\| & \| \\
\bigcup_{x \in X} \sigma_{R S}(x) \cup \mathcal{S}(R S)=\bigcup_{x \in X} \sigma_{S R}(S x) \cup \mathcal{S}(S R) \subset \bigcup_{y \in Y} \sigma_{S R}(y) \cup \mathcal{S}(S R)
\end{array}
$$

Now from $R$ injective it follows that $\sigma(S R) \subset \sigma(R S)$ and hence 1. is proved.

2. and 3. are obtained similarly.

4. As in 1. we have $\sigma(S R) \subset \sigma(R S)$ and since $S$ is dense range, $S^{*}$ is one to one. It follows that $\sigma(R S)=\sigma\left(S^{*} R^{*}\right) \subset \sigma\left(R^{*} S^{*}\right)=\sigma(S R)$.

Remarks. 1) The situation where $R$ or $S$ is normal is derived as follows. Suppose $S$ is normal, If $S$ is not one to one, then $S^{*}$ is also not one to one and we apply (2) in the previous corollary and $0 \in \sigma_{p}\left(R^{*} S^{*}\right) \subset \sigma\left(R^{*} S^{*}\right)=\sigma(R S)$. Otherwise, $S$ is injective with dense range and hence the equality is given by (4).

2) The normality in the previous assumption cannot be relaxed to subnormality as is shown by the case when $S$ is the usual shift and $R=S^{*}$ its adjoint.

Let $F$ be a subset of the complex plane and $T \in \mathcal{L}(X)$, the local analytic spectral subspace associated with $F$ is given by $X_{T}(F)=\left\{x \in X: \sigma_{T}(x) \subseteq F\right\}$. Obviously if $T$ has the $S V E P$, then $X_{T}(F)=\mathcal{X}_{T}(F)$. We have

Corollary 3.2. Let $F$ be a subset of the complex plane. Then

i) $S\left(X_{R S}(F)\right) \subset Y_{S R}(F) \cap S(X)$ and $S\left(\mathcal{X}_{R S}(F)\right) \subset \mathcal{Y}_{S R}(F) \cap S(X)$ (resp. $R\left(Y_{S R}(F)\right)$ $\subset X_{R S}(F) \cap R(Y)$ and $\left.R\left(\mathcal{Y}_{S R}(F)\right) \subset \mathcal{X}_{R S}(F) \cap R(X)\right)$.

ii) If $S$ is one to one, we get

$$
S\left(X_{R S}(F)\right)=Y_{S R}(F) \cap S(X) \quad \text { and } \quad S\left(\mathcal{X}_{R S}(F)\right)=\mathcal{Y}_{S R}(F) \cap S(X) .
$$

As a direct consequence of Proposition 3.1, we have

Corollary 3.3. If $R$ and $S$ are dense range, then $R S$ has the decomposition property $\left(\delta_{w}\right)$ if and only if $S R$ has the decomposition property $\left(\delta_{w}\right)$

Recall that $T$ is said to have the fat local spectra property if $\sigma_{T}(x)=\sigma(T)$ for every $x \neq 0$. We have the following result.

Corollary 3.4. If $R$ and $S$ are one to one, then

$R S$ has fat local spectra property if and only if $S R$ has fat local spectra property. 
Let $r($.$) be the spectral radius function defined on operators. It is obvious that$ $r(R S)=r(S R)$ for arbitrary $R$ and $S$. This turns out to be true for local spectral radii. For $x \in X$ and $T \in \mathcal{L}(X)$, the local spectral radius at $x$ is defined to be $r_{T}(x)=$ $\limsup _{n \rightarrow \infty}\left\|T^{n} x\right\|^{1 / n}$, then we have

Remark. Again if $R$ or $S$ is not one to one, the previous corollary fails. To see this, take $S$ to be the usual shift on the Hardy space and $R=S^{*}$ to be its adjoint.

Proposition 3.2. For every $x \in X$,

$$
r_{R S}(x)=r_{S R}(S x) .
$$

Proof. Derives from the observation

$$
\left\|(R S)^{n} x\right\| \leq\|R\|\left\|(S R)^{n-1} S(x)\right\| \leq\|R\|\|S\|\left\|(R S)^{n-1} x\right\| .
$$

The quasi-nilpotent part of $T$ usually denoted by $X_{0}(T)$ is the set of all elements $x \in X$ such that $r_{T}(x)=0$. The analytic core $K(T)$ of $T$ is the set of all elements $x \in H$ such that there exists a sequence $\left(u_{n}\right)_{n \in \mathbb{N}} \subset X$ and $c>0$ for which $x=u_{0}, T u_{n+1}=u_{n}$ and $\left\|u_{n}\right\| \leq c^{n}$. The introduction of $X_{0}(T)$ and $K(T)$ goes back to M. Mbekhta in [23], see also [24] for further details and results. It is not difficult to see that $X_{0}(T)=\mathcal{X}_{T}(\{0\})$ and $K(T)=X_{T}(\mathbb{C} \backslash\{0\})$. The operator $T$ is said to have trivial quasi-nilpotent part (resp. trivial analytic core) if $X_{0}(T)=\{0\}$ (resp. $\left.K(T)=\{0\}\right)$.

The following result is immediate from Corollary 3.2:

COROllary 3.5. Under the assumptions of Corollary 3.4, we have

(1) $S R$ has trivial quasi-nilpotent part if and only if $R S$ has trivial quasi-nilpotent part.

(2) $S R$ has trivial analytic core if and only if $R S$ has trivial analytic core.

\section{Applications}

4.1. Aluthge transforms. Let $T \in \mathcal{L}(H)$ be a bounded operator on some Hilbert space $H$ and $U|T|$ be its polar decomposition, where $|T|=\left(T^{*} T\right)^{\frac{1}{2}}$ and $U$ is the appropriate partial isometry. The Aluthge transform of $T$ is given by $\tilde{T}=|T|^{\frac{1}{2}} U|T|^{\frac{1}{2}}$ and was first considered by A. Aluthge to extend some inequalities related to hyponormality. To be precise:

Recall that an operator $A \in \mathcal{L}(H)$ is said to be $p$-hyponormal $(p>0)$ if $\left(A^{*} A\right)^{p} \geq$ $\left(A A^{*}\right)^{p}$ (i.e. $|A|^{2 p} \geq\left|A^{*}\right|^{2 p}$ ) and $T$ is $\log$-hyponormal if $\log \left(A^{*} A\right) \geq \log \left(A A^{*}\right)$. A 1 hyponormal operator is a hyponormal operator and $\frac{1}{2}$-hyponormal operators are called semi-hyponormal ([3, 29]). The Löwner-Heinz inequality implies that if $A$ is $q$-hyponormal then it is $p$-hyponormal for any $0<p \leq q$. An invertible operator $A$ is said to be loghyponormal $([28])$ if $\log \left(A^{*} A\right) \geq \log \left(A A^{*}\right)$.

It is known that if $T$ is $p$-hyponormal $(p>0)$, then $\tilde{T}$ is $p+\frac{1}{2}$-hyponormal and $\tilde{T}$ is hence hyponormal. If $T$ is log-hyponormal, then $\tilde{\tilde{T}}$ is semihyponormal. $T$ is said to be w-hyponormal ([4]) if $|\tilde{T}| \geq|T| \geq\left|\tilde{T}^{*}\right|$, so $\tilde{T}$ is semi-hyponormal if $T$ is w-hyponormal. It is also known ([4]) that $p$-hyponormal $(p>0)$ and log-hyponormal operators are w-hyponormal. 
Since then this concept received a lot of interest by numerous mathematicians. In a series of papers $[18,19,20]$ I. B. Jung, E. Ko and C. Pearcy have investigated common spectral properties of $\tilde{T}$ and $T$. It is shown, once again, that $\tilde{T}$ and $T$ share most of their spectral properties.

Set $S=|T|^{\frac{1}{2}}$ and $R=U|T|^{\frac{1}{2}}$. Then clearly $R S=T$ and $S R=\tilde{T}$. In particular $\tilde{T}$ and $T$ have almost the same local spectral properties.

Proposition 4.1. Let $T$ be a bounded operator on some Hilbert space and let $\tilde{T}$ be its Aluthge transform. Then T has the SVEP (resp. T has Bishop's property $\beta$, resp. T has property $(\delta)$, resp. $T$ is decomposable, is subscalar) if and only if $\tilde{T}$ has the SVEP, (resp. $\tilde{T}$ has Bishop's property $\beta$, resp. $\tilde{T}$ has property $(\delta)$, resp. $\tilde{T}$ is decomposable, is subscalar).

Since $T$ is one to one if and only if $\tilde{T}$ is one to one, we get from Corollary 3.1

Corollary 4.1. $\sigma(T)=\sigma(\tilde{T})$.

Consider $T \in \mathcal{L}(H)$ and let $U|T|$ be its polar decomposition, and for $s, t \geq 0, T(s, t)=$ $|T|^{s} U|T|^{t}$ is called the generalized Aluthge transform of $T$. Remark that $\tilde{T}=T\left(\frac{1}{2}, \frac{1}{2}\right)$.

Take $R=|T|^{r}$ and $S=|T|^{s} U|T|^{t-r}$. Then $S R=T(s, t)$ and $R S=T(s+r, t-r)$. Thus, we have the following result:

Corollary 4.2. Let $T \in \mathcal{L}(H), s \geq 0$ and $0<r<t$, then $T(s, t)$ has the property $(\beta)$ (respectively, $(\delta)$ or is subscalar) if and only if $T(s+r, t-r)$ has the property $(\beta)$ (respectively, $(\delta)$ or is subscalar). In particular, $T:=T(0,1) \in(\beta)$ (respectively, $(\delta)$ or is subscalar) if and only if $T(r, 1-r)(0<r<1)$ has the property $(\beta)$ (respectively, $(\delta)$ or is subscalar).

The previous result appears in [9] under the restrictive assumption where $N(T) \subset$ $N\left(T^{*}\right)$.

We also deduce

Corollary 4.3. Let $T \in \mathcal{L}(H)$ be -hyponormal, log-hyponormal or $w$-hyonormal, then $T$ is subscalar.

4.2. Spectral picture and Weyl's theorem. Denote by $\mathcal{F}$ (resp. $\mathcal{S} F, \mathcal{L} F$ and $\mathcal{R} F$ ) the family of all Fredholm (resp. semi-Fredholm, left Fredholm and right Fredholm) operators. The essential spectrum of $T$ is $\sigma_{e}(T)=\{\lambda \in \mathbb{C}: T-\lambda \notin \mathcal{F}\}$. The semi-Fredholm spectrum $\sigma_{s e}(T)$, the left essential spectrum $\sigma_{l e}(T)$ and the right essential spectrum $\sigma_{r e}(T)$ are defined similarly. The Weyl spectrum, $\sigma_{w}(T)$, is the set of all $\lambda \in \mathbb{C}$ such that $T-\lambda$ is not Fredholm with index zero.

It is well known that the mapping ind $: \mathcal{S} F \rightarrow \mathbb{Z} \cup\{\infty,+\infty\}$ is continuous. We shall call a hole in $\sigma_{e}(T)$ any bounded component of $\mathbb{C} \backslash \sigma_{e}(T)$ and a pseudo hole a component of $\sigma_{e}(T) \backslash \sigma_{l e}(T)$ or $\sigma_{e}(T) \backslash \sigma_{r e}(T)$. The spectral picture $S P(T)$ of an operator was introduced by C. Pearcy in [26] as the collection of holes and pseudo holes in $\sigma_{e}(T)$ and the associated Fredholm indices. Since for every $\lambda \neq 0, R(R S-\lambda)$ is closed if and only if $R(S R-\lambda)$ is closed and since obviously $\operatorname{dim}(N(R S-\lambda))=\operatorname{dim}(N(S R-\lambda))$, one concludes easily that $R S$ and $S R$ have the same spectral picture. Thus in section $4.2, T$ and $\tilde{T}$ have the same spectral picture as proved in [19]. 
For $T$ a bounded operator, we write $i s o \sigma(T)$ for the set of all isolated points of $\sigma(T)$, and we define

$$
\Pi_{00}(T)=\{\lambda \in \operatorname{iso} \sigma(T): 0<\operatorname{dim}(N(T-\lambda))<\infty\} .
$$

An operator is said to satisfy Weyl's theorem if

$$
\sigma_{w}(T)=\sigma(T) \backslash \Pi_{00}(T)
$$

From the discussion above, it follows that $\Pi_{00}(R S) \backslash\{0\}=\Pi_{00}(S R) \backslash\{0\}$ and that $\sigma_{w}(R S) \backslash\{0\}=\sigma_{w}(S R) \backslash\{0\}$. Thus

Proposition 4.2. If $0 \in \Pi_{00}(S R) \cap \Pi_{00}(R S)$ or $0 \notin \Pi_{00}(S R) \cup \Pi_{00}(R S)$, then $R S$ satisfies Weyl's theorem if and only if $S R$ satisfies Weyl's theorem.

The preceding Proposition and Corollary 3.1 allow us to get

COROLlary 4.4. If $R$ and $S$ are injective, then $R S$ satisfies Weyl's theorem if and only if $S R$ satisfies Weyl's theorem.

It is not hard to see that $\sigma_{p}(T)=\sigma_{p}(\tilde{T})$ and hence

COROLlary 4.5. Let $T$ be a bounded operator on some Hilbert space and let $\tilde{T}$ be its Aluthge transform. Then, $T$ satisfies Weyl's theorem if and only if $\tilde{T}$ satisfies Weyl's theorem.

We finally note that the equivalence in Corollary 4.5 does not hold in general. Indeed, let $S$ be the unilateral shift on the Hardy space and $R=S^{*}$ its adjoint operator. Then $R S$ is the identity operator and hence satisfies clearly Weyl's theorem, while for $S R$, we have

$$
\sigma_{w}(S R)=\{0,1\} \neq \sigma(S R) \backslash \Pi_{00}(S R)=\{1\} .
$$

4.3. Applications to operator matrices. Let $A \in \mathcal{L}(X), B \in \mathcal{L}(Y)$ and $C \in \mathcal{L}(Y, X)$. We consider $M_{C}$ as the upper triangular $2 \times 2$ matrix $\left[\begin{array}{cc}A & C \\ 0 & B\end{array}\right]$. The inclusion $\sigma\left(M_{C}\right) \subset$ $\sigma(A) \cup \sigma(A)$ is trivial, and the equality fails generally. Numerous papers were devoted to the last problem, see $[6,12,17,13,31]$ for example.

An auxiliary problem is: Given $A$ and $B$, for which operators $C$ do we have

$$
\sigma\left(M_{C}\right)=\sigma(A) \cup \sigma(A) ?
$$

For a bounded operator $T$, let $N^{\infty}(T)=\cup_{n \geq 1} N\left(T^{n}\right)$ denote the generalized kernel of $T$. Let also $L_{T}, R_{T}$ and $\delta_{T}$ be the left multiplication by $T$, the right multiplication by $T$ and the inner derivation associated with $T$, that is,

$L_{T}(X)=T X, R_{T}(X)=X T$ and $\delta_{T}(X)=T X-X T$ for any bounded operator $X$.

We apply the main results of this paper to prove some stability of spectral properties on upper triangular matrices in the line of [6].

Theorem 4.1. Let $A \in \mathcal{L}(X), B \in \mathcal{L}(Y)$ be given and $\Sigma \in\left\{\sigma_{\beta}, \sigma_{\beta_{\epsilon}}, \sigma_{\delta}, \sigma_{\text {dec }}\right\}$. Then for every $C \in \operatorname{cl}\left[R\left(\delta_{A, B}\right)+N\left(\delta_{A, B}\right)+\bigcup_{\lambda \in \mathbb{C}} N^{\infty}\left(L_{A-\lambda}\right)+\bigcup_{\lambda \in \mathbb{C}} N^{\infty}\left(R_{B-\lambda}\right)\right]$, we have

$$
\Sigma\left(M_{C}\right)=\Sigma\left(M_{0}\right) \text {. }
$$

TheOrem 4.2. Let $A \in \mathcal{L}(X), B \in \mathcal{L}(Y)$ be bounded operators and $C \in \operatorname{cl}\left[R\left(\delta_{A, B}\right)+\right.$ $\left.N\left(\delta_{A, B}\right)+\bigcup_{\lambda \in \mathbb{C}} N^{\infty}\left(L_{A-\lambda}\right)+\bigcup_{\lambda \in \mathbb{C}} N^{\infty}\left(R_{B-\lambda}\right)\right]$. The following hold: 
(1) If $\Sigma \in\left\{\sigma, \sigma_{e}, \sigma_{w}\right\}$, then $\Sigma\left(M_{C}\right)=\Sigma\left(M_{0}\right)$.

(2) If $\Sigma \in\left\{\sigma_{a p}, \sigma_{s}, \sigma_{S F}\right\}$, then $\Sigma_{*}\left(M_{C}\right)=\Sigma_{*}\left(M_{0}\right)$ where $\Sigma_{*}()=.\Sigma(.) \backslash\{0\}$.

(3) If moreover, $C \in \operatorname{cl}\left[R\left(\delta_{A, B}\right)+N\left(\delta_{A, B}\right)+\bigcup_{\lambda, \mu \in \mathbb{C}}\left(N^{\infty}\left(L_{A-\lambda}\right) \cap N^{\infty}\left(R_{B-\mu}\right)\right)\right]$, then $\Sigma\left(M_{C}\right)=\Sigma\left(M_{0}\right)$ for $\Sigma \in\left\{\sigma_{a p}, \sigma_{s}, \sigma_{S F}\right\}$.

The proof of the previous theorem can be found in [8] and relies heavily on the next lemma.

LEMMA 4.1. Let $T$ be a bounded operator and $N$ a commuting operator with $T$ such that $N^{2}=0$. Then

$$
\Sigma(T+N)=\Sigma(T)
$$

for $\Sigma \in\left\{\sigma, \sigma_{e}, \sigma_{w}, \sigma_{S F}, \sigma_{a p}, \sigma_{s}, \mathcal{S}, \sigma_{\beta}, \sigma_{\beta_{\epsilon}}, \sigma_{\delta}, \sigma_{d e c}\right\}$.

REMARK 4.1. 1) It has been shown in [8] that $\Sigma_{*}\left(M_{C}\right)=\Sigma_{*}\left(M_{0}\right)$ for a large family of spectra. We notice that from $[6,31], \Sigma_{*}\left(M_{0}\right) \backslash \Sigma_{*}\left(M_{C}\right)$ cannot be a singleton (for example if we deal with $\sigma, \sigma_{e}, \sigma_{w}$, the difference is an open set). We deduce that for such spectra, we have $\Sigma\left(M_{C}\right)=\Sigma\left(M_{0}\right)$.

2) In theorem $4.2(2)$, it is not generally possible to get $\Sigma\left(M_{C}\right)=\Sigma\left(M_{0}\right)$. The purpose of the following example is to show this fact.

ExAmple 4.1. Let $A=S$ be the shift operator on the Hardy space $H^{2}, B=I-e_{0} \otimes e_{0}$ and $C=e_{0} \otimes e_{0}$ with $e_{0}$ is the first element of the canonical orthonormal basis of $H^{2}$. Then clearly $C B=0$ and $\sigma_{a p}\left(M_{0}\right)=\{z \in \mathbb{C}:|z|=1\} \cup\{0\}$. Let us prove that $0 \notin \sigma_{a p}\left(M_{C}\right)$. Suppose that for some $\left(x_{n}, y_{n}\right)$, we have $\lim _{n \rightarrow \infty} M_{C}\left(x_{n}, y_{n}\right)=0$. Then

$$
\left\{\begin{array}{l}
\lim _{n \rightarrow \infty}\left(S x_{n}+e_{0} \otimes e_{0}\left(y_{n}\right)\right)=0 \\
\lim _{n \rightarrow \infty}\left(I-e_{0} \otimes e_{0}\right) y_{n}=0 .
\end{array}\right.
$$

Now since $R(S) \perp \mathbb{C}_{0}$, we deduce that $\lim _{n \rightarrow \infty}\left(S x_{n}\right)=\lim _{n \rightarrow \infty}\left(e_{0} \otimes e_{0}\right) y_{n}=0$. Then $\lim _{n \rightarrow \infty} y_{n}=\lim _{n \rightarrow \infty}\left(e_{0} \otimes e_{0}\right) y_{n}+\lim _{n \rightarrow \infty}\left(I-e_{0} \otimes e_{0}\right) y_{n}=0$ and $\lim _{n \rightarrow \infty} x_{n}=$ $\lim _{n \rightarrow \infty} S^{*} S x_{n}=0$.

4.4. Weyl's theorem for operator matrices. Let $A$ and $B$ be given, in general the fact that Weyl's theorem holds for $M_{0}(=A \oplus B)$ does not imply that Weyl's theorem holds for $M_{C}$, see for instance [21]. It also may happen that $M_{C}$ satisfies Weyl's theorem while $M_{0}$ does not satisfy it. We consider the following example to show the last claim. If $A=B^{*}$ is the shift operator and $C=I-A B$, then $M_{C}$ is unitary without eigenvalues and hence satisfies Weyl's theorem. Now $\sigma_{w}\left(M_{0}\right)=\{z:|z|=1\}$ and $\sigma\left(M_{0}\right) \backslash \pi_{00}\left(M_{0}\right)=\bar{D}$. Thus $\sigma_{w}\left(M_{0}\right)$ does not satisfy Weyl's theorem.

By [7, Proposition 4.4] and Theorem 4.2 we get

Proposition 4.3. Let $A, B$ be given and suppose that $0 \in \Pi_{00}\left(M_{C}\right) \cap \Pi_{00}\left(M_{0}\right)$ or $0 \notin \Pi_{00}\left(M_{C}\right) \cup \Pi_{00}\left(M_{0}\right)$, for some $C \in \operatorname{cl}\left[R\left(\delta_{A, B}\right)+N\left(\delta_{A, B}\right)+\bigcup_{\lambda \in \mathbb{C}} N^{\infty}\left(L_{A-\lambda}\right)+\right.$ $\left.\bigcup_{\lambda \in \mathbb{C}} N^{\infty}\left(R_{B-\lambda}\right)\right]$. Then Weyl's theorem holds for $M_{0}$ if and only if Weyl's theorem holds for $M_{C}$.

Proof. For the obvious symmetry reason we shall show only one way. In [7, Proposition 4.4], it is shown that if $0 \in \pi_{00}(R S) \cap \pi_{00}(S R)$ or $0 \notin \pi_{00}(R S) \cup \pi_{00}(S R)$ then Weyl's theorem holds for $R S$ if and only if Weyl's theorem holds for $S R$. Now if Weyl's theorem 
holds for $A \oplus B$, we argue as in Step 1 of the proof of Theorem 4.2 to see that Weyl's theorem holds for $M_{C_{2}+C_{1}}$, when $C_{2}+C_{1} \in \bigcup_{\lambda \in \mathbb{C}} N^{\infty}\left(L_{A-\lambda}\right)+\bigcup_{\lambda \in \mathbb{C}} N^{\infty}\left(R_{B-\lambda}\right)$. Now, since $M_{C_{3}+C_{2}+C_{1}}=M_{C_{2}+C_{1}}+N$, with $N=\left[\begin{array}{cc}0 & C_{3} \\ 0 & 0\end{array}\right]$ nilpotent and $N M_{C_{2}+C_{1}}=M_{C_{2}+C_{1}} N$, for $C_{3} \in N\left(\delta_{A, B}\right)$, it follows from [25, Theorem 3] that Weyl's theorem holds for $M_{C_{3}+C_{2}+C_{1}}$. Finally $M_{C_{4}+C_{3}+C_{2}+C_{1}}$ and $M_{C_{3}+C_{2}+C_{1}}$ are similar, for $C_{4} \in R\left(\delta_{A, B}\right)$ yields Weyl's theorem for $M_{C}$.

Acknowledgements. We would like to thank for the support of l'Action intégrée francomarocaine (no. MA/03/64).

\section{References}

[1] E. Albrecht, An example of a weakly decomposable operator, which is not decomposable, Rev. Roumaine Math. Pures Appl. 20 (1975), 855-861.

[2] E. Albrecht and J. Eschmeier, Analytic functional models and local spectral theory, Proc. London Math. Soc. 75 (1997), 323-348.

[3] A. Aluthge, On p-hyponormal operators for $0<p<1$, Integral Equations Operator Theory 13 (1990), 307-315.

[4] A. Aluthge and D. Wang, w-hyponormal operators II, Integral Equations Operator Theory 37 (2000), 324-331.

[5] B. Barnes, Common operator properties of the linear operators RS and SR, Proc. Amer. Math. Soc. 126 (1998), 1055-1061.

[6] M. Barraa and M. Boumazgour, A note on the spectrum of an upper triangular operator matrix, Proc. Amer. Math. Soc. 131 (2003), 3083-3088.

[7] C. Benhida and E. H. Zerouali, Local spectral theory of linear operators $R S$ and $S R$, Integral Equations Operator Theory 54 (2006), 1-8.

[8] C. Benhida, E. H. Zerouali and H. Zguitti, Spectra of upper triangular operator matrices, Proc. Amer. Math. Soc. 133 (2005), 3013-3020.

[9] Lin Chen, Yan Zikun and Ruan Yingbin, p-Hyponormal operators are subscalar, Proc. Amer. Math. Soc. 131 (2003), 2753-2759.

[10] Lin Chen, Yan Zikun and Ruan Yingbin, Common operator properties of operators $R S$ and $S R$ and p-hyponormal operators, Integral Equations Operator Theory 43 (2002), 313-325.

[11] M. Chō, On spectra of $A B$ and $B A$, Proc. KOTAC 3 (2000), 15-19.

[12] H. K. Du and J. Pan, Perturbation of spectrums of $2 \times 2$ operator matrices, Proc. Amer. Math. Soc. 121 (1994), 761-776.

[13] H. Elbjaoui and E. H. Zerouali, Local spectral theory for $2 \times 2$ operator matrices, Int. J. Math. and Mathematical Sciences 42 (2003), 2667-2672.

[14] J. K. Finch, The single valued extension property on a Banach space, Pacific J. Math. 58 (1975), 61-69.

[15] J. K. Han, H. Y. Lee and W. Y. Lee, Invertible completion of $2 \times 2$ upper triangular operator matrices, Proc. Amer. Math. Soc. 128 (1999), 117-132.

[16] D. A. Herrero, Approximation of Hilbert Space Operators, Vol. 1, Pitman Research Notes in Mathematics Series 224, New York, 1989.

[17] M. Houimdi and H. Zguitti, Propriétés spectrales locales d'une matrice carrée des opérateurs, Acta Math. Vietnam. 25 (2000), 137-144. 
[18] I. B. Jung, E. Ko and C. Pearcy, Aluthge transforms of operators, Integral Equations Operator Theory 37 (2000), 437-448.

[19] I. B. Jung, E. Ko and C. Pearcy, Spectral picture of Aluthge transforms of operators, Integral Equations Operator Theory 40 (2001), 52-60.

[20] I. B. Jung, E. Ko and C. Pearcy, The iterated Aluthge transforms, Integral Equations Operator Theory 45 (2003), 375-387.

[21] W. Y. Lee, Weyl spectra for operator matrices, Proc. Amer. Math. Soc. 129 (2000), 131138.

[22] K. B. Laursen and M. M. Neumann, An Introduction to Local Spectral Theory, London Mathematical Society Monogr. 20 (2000).

[23] M. Mbekhta, Resolvant généralisé et théorie spectrale, Journal of Operator Theory 21 (1989), 69-105.

[24] M. Mbekhta and A. Ouahab, Opérateur s-régulier dans un espace de Banach et théorie spectrale, Acta Sci. Math. (Szeged) 59 (1994), 525-543.

[25] K. K. Oberai, On the Weyl's spectrum (II), Illinois J. Math. 21 (1977), 84-90.

[26] C. Pearcy, Some Recent Developments in Operator Theory, CBMS Regional Conference Ser. in Mathematics 36, Amer. Math. Soc., Providence, 1978.

[27] M. Schechter, Riesz operators and Fredholm perturbations, Bull. Amer. Math. Soc. 74 (1968), 1139-1144.

[28] K. Tanahashi, On log-hyponormal operators, Integral Equations Operator Theory 34 (1999), 364-372.

[29] D. Xia, Spectral Theory of Hyponormal Operators, Birkhäuser Verlag, Basel, 1983.

[30] E. H. Zerouali and H. Zguitti, On the weak decomposition property ( $\delta)$, Studia Math. 167 (2005), 17-28.

[31] E. H. Zerouali and H. Zguitti, Perturbation of spectra of operator matrices and local spectral theory, preprint. 